\title{
Minimization of fuel consumption through optimum material selection of a car frame production company
}

\author{
R. Eleni \& A. Panagiotis \\ Department of Business Administration, Aegean University, Greece
}

\begin{abstract}
In the last decade it has been recognized that urban environmental pollution is strongly influenced by car fuel consumption. As the innovations in the motor technology have already been saturated, the interest is focused on the selection of the suitable materials in order to minimize car weight without loss on stiffness and safety. However, materials leading to light-weight cars, and therefore fuel consumption economy, are often expensive. The balancing between the unit cost and the mass saving of the material (without loss of the product quality) is the criterion for the decision making for optimum material selection. Therefore, a simple model correlating technical and financial data has been developed to classify the candidate materials in order to make the optimum decision concerning selection of the material which results in the minimum cost of a lightweight car, and therefore the minimum exhaust-gas emissions. The present analysis indicated that CFRPs are the most appropriate materials which result in the production of low cost and low fuel consumption cars. However, these materials cause environmental damage due to their inability to be recycled. The balance between environmental cost benefits and losses by using CFRPs is not clearly obvious and it will be the subject of further work.
\end{abstract}

Keywords: fuel consumption, cost-benefit analysis, material selection, cost minimization, Carbon Fiber Reinforced Polymers.

\section{Introduction}

To increase car competitiveness through the minimization of its fuel consumption two solutions can be followed: (a) to improve the motor efficiency and (b) to reduce the weight of the car. 
The reduction of fuel consumption, following the first solution, is limited because existing motor technology has already produced highly efficient engines. As fuel consumption can be considered to be proportional [1] to the weight of the car (fig. 1), the second route seems to be more realistic. However, the weight of a car is influenced by the mechanical and physical properties of the selected materials while the cost depends on the weight saving and the material unit price. The above balance should be performed on the basis of constant product quality.

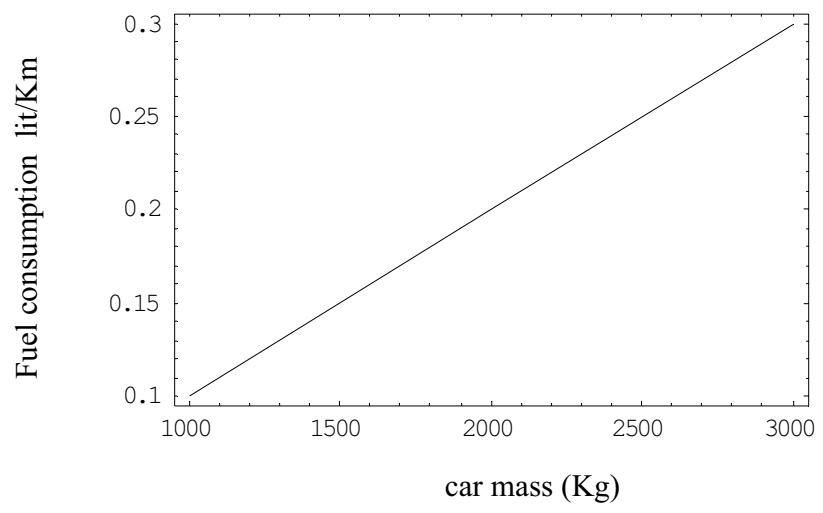

Figure 1: Diagram of fuel consumption vs mass of car, derived by real specifications of nine car types.

In the present work, a parametric study is performed in order to support the optimum decision for the suitable material selection for a car frame with the lowest weight of cost with the same stiffness or the same strength.

To this scope, a simple model for the determination of the dependency of the car frame mass by the mechanical properties and the material cost will be firstly developed in order to derive a table with the suitable data for decision making (e.g. [2-5]).

\section{Techno-economical model}

As the mass of the body makes up $60-70 \%$ of the total mass of the vehicle, the biggest weight saving can be achieved by material replacement of the car frame. Candidate materials due to the differences in their physical and mechanical properties should have differences in the structural part dimensions and consequently in the corresponding total mass in order to achieve a structurally equivalent product. To satisfy this criterion the weight of a simplified structural car frame model will be associated with the material properties and the total cost in order to achieve (a) a frame with the same stiffness or (b) a frame with the same strength. 


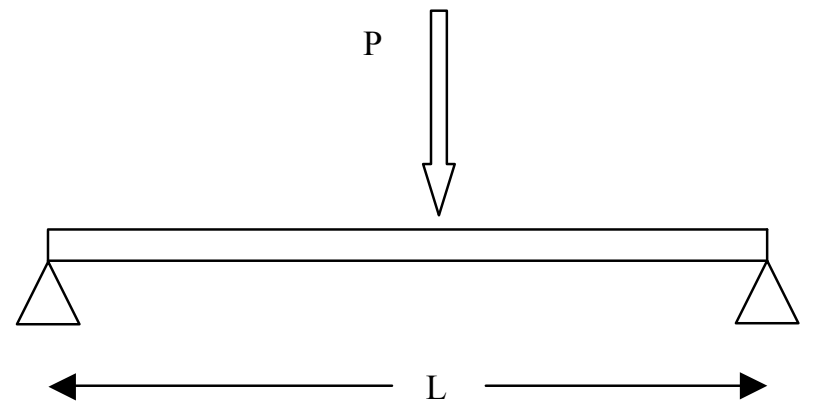

Figure 2: Deflection of a simulated car frame.

\subsection{Constant frame stiffness}

A car frame is considered to be simulated by a simply supported beam with length $\mathrm{L}$, cross section area $\mathrm{A}$, moment of inertia I, density $\rho$, modulus of elasticity $E$, and strength $S_{u}$. The maximum elastic deflection $f$ due to a force $P$ acting in the middle of the model can be obtained by the Castigliano theorem [6].

$$
f=\frac{\partial U}{\partial P}
$$

where $U$ is the accumulated elastic energy of the beam.

It is well known [6] that

$$
U=\frac{1}{2 E I} \int_{L} M^{2}(x) d x
$$

where $\mathrm{M}(\mathrm{x})$ is the bending moment distribution of the beam given by the following function [6]:

$$
M(x)=\frac{1}{2} P x
$$

Then, eqn (2) takes the form:

$$
U=2 \frac{P^{2}}{4 E I} \int_{0}^{L / 2} x^{2} d x
$$

or

$$
U=\frac{P^{2} L^{3}}{48 E I}
$$


192 Environmental Economics and Investment Assessment

Considering eqn (5), eqn (1) can be written:

$$
f=\frac{P L^{3}}{24 E I}
$$

For a rectangular cross section with dimensions $\mathrm{b} \times \mathrm{h}$ the moment of inertia I takes [6] the value:

$$
I=\frac{b h^{3}}{12}
$$

Then eqn (6) takes the form:

$$
f=\frac{P L^{3}}{2 E b h^{3}}
$$

The corresponding mass of the selected beam is

$$
m=\rho A L
$$

where A is the cross section area. Then

$$
m=\rho b h L
$$

The quantity h of the cross section can be obtained by eqn (8)

$$
h=L \sqrt[3]{\frac{P}{2 f E b}}
$$

Considering eqn (11), eqn (10) results

$$
m=b L^{2} \sqrt[3]{\frac{1}{2 b}\left(\frac{P}{f}\right)} \frac{\rho}{\sqrt[3]{E}}
$$

For a given stiffness $(\mathrm{P} / \mathrm{f})$ and bar dimensions $\mathrm{b}, \mathrm{L}$ it is concluded that the weight of the car frame is dependent on the quantity $\rho / \sqrt[3]{E}$.

\subsection{Constant frame strength}

The maximum applied stress $\sigma_{\max }$ in the simple model of fig. 2 can be obtained [6] by the following equation:

$$
\sigma_{\max }=\frac{M_{\max }}{2 I} h
$$


where $\mathrm{M}_{\max }$ is the maximum bending moment given by

$$
M_{\text {max }}=\frac{P}{2} \frac{L}{2}
$$

Taking into account eqns (7) and (14), eqn (13) can be written:

$$
\sigma_{\max }=\frac{3 P L}{2 b h^{2}}
$$

The panel of fig. 2 failed at stress

$$
\sigma_{\max }=S_{u}
$$

where $S_{u}$ is the strength of the material. Then, according to eqns (15) and (16) it can be written:

$$
h=\sqrt{\frac{3 P L}{2 b S_{u}}}
$$

and the mass of the panel can be derived by eqn (10):

$$
m=\frac{b L \sqrt{3 P L}}{\sqrt{2 b}} \frac{\rho}{\sqrt{S_{u}}}
$$

Eqn. (18) shows that the weight of the car frame depends on the quantity $\rho / \sqrt{S_{u}}$.

\section{Parametric study and decision making}

To support the decision making concerning the suitable material which results in the light-weight car frame with the lowest cost, Table 1 containing the candidate materials with the corresponding mechanical properties, prices and the derived classification indexes is formulated.

The index N.M. denotes the normalized mass of the material $i$ with respect to mild steel obtained by the equation

$$
N . M .=\frac{\rho_{i} / \sqrt[3]{E_{i}}}{\rho_{s t} / \sqrt[3]{E_{s t}}}
$$

for classification with respect to the stiffness, and

$$
N . M .=\frac{\rho_{i} / \sqrt{S u_{i}}}{\rho_{s t} / \sqrt{S u_{s t}}}
$$

for classification with respect to the strength. 
Table 1: Results for the decision support for the cost effective material.

\begin{tabular}{|c|c|c|c|c|c|c|c|c|c|c|}
\hline & \multirow{2}{*}{$\boldsymbol{\rho}_{\operatorname{ton} / \mathbf{m}^{3}}$} & \multirow{2}{*}{\begin{tabular}{|l|} 
Su \\
MPa
\end{tabular}} & \multirow{2}{*}{\begin{tabular}{|l|} 
E \\
GPa
\end{tabular}} & \multirow{2}{*}{$\rho / \sqrt[3]{E}$} & \multirow{2}{*}{$\rho / \sqrt{S u}$} & \multicolumn{2}{|c|}{ N.M. } & \multirow[b]{2}{*}{$\begin{array}{l}\text { Unit } \\
\text { price } \\
\text { Euro/ } \\
\text { Kg }\end{array}$} & \multicolumn{2}{|c|}{ N.C. } \\
\hline & & & & & & Stiffness & Strength & & Stiffness & Strength \\
\hline Mild steel & 7.8 & 430 & 207 & 1.32 & 0.38 & 1.00 & 1.00 & 0.65 & 0.65 & 0.65 \\
\hline \begin{tabular}{|l|} 
Aluminum \\
\end{tabular} & 2.7 & 200 & 69 & 0.66 & 0.19 & 0.50 & 0.50 & 193 & 96.5 & 96.5 \\
\hline \begin{tabular}{|l|} 
Cooper \\
alloys
\end{tabular} & 9.0 & 625 & 150 & 1.69 & 0.36 & 1.28 & 0.94 & 350 & 448 & 329 \\
\hline Nickel & 8.9 & 400 & 214 & 1.49 & 0.44 & 1.13 & 1.16 & 12 & 13.56 & 13.9 \\
\hline \begin{tabular}{|l|} 
CFRPs \\
(Carbon \\
Fiber \\
Reinforced \\
Polymers) \\
\end{tabular} & 1.6 & 655 & 200 & 0.27 & 0.06 & 0.20 & 0.16 & 0.25 & 0.05 & 0.04 \\
\hline
\end{tabular}

To compare the mass saving with the material unit cost, the index of the normalized cost

$$
\text { N.C. }=(N . M .) x(\text { material unit price })
$$

is derived in order to classify the candidate materials with respect to their cost.

By the columns containing the values N.M. and N.C. the following observations can be derived:

(a) The CFRPs combine very low density resulting to the minimum values of $\rho / \sqrt[3]{E}, \rho / \sqrt{S u}$ as well as low unit price. Therefore, these materials lead to the most cost effective light-weight car frames with the same stiffness and strength as the other candidate materials. More specifically, the CFRPs provide $(1-0.20) \times 100 \%=80 \%$ lower mass than steel with respect to the stiffness and $(1-0.16) \times 100 \%=84 \%$ lower mass than steel with respect to the strength. The corresponding indexes concerning the cost saving in comparison with the steel are $(0.65-0.05) \times 100 \%=60 \%$ with respect to the stiffness and $(0.65-0.04) \times 100 \%=61 \%$ with respect to the strength. The above results also confirm the rapid increase of the use of the CFRPs in the aerospace industry where mass saving is the main design criterion.

(b) Despite the fact that aluminum is lighter (N.M. $=0.5)$ than the other candidate materials (except CFRPs) with respect to both stiffness and strength, however, concerning the material cost, it is classified in lower rank than steel and nickel because of its much higher unit price.

(c) The current widely-used mild steel takes third rank (with respect to both stiffness and strength) concerning the normalized mass but its normalized cost is lower than the other candidate materials (except CFRPs) because of its very low price.

(d) Copper alloy due to higher density $\left(9.0 \mathrm{tn} / \mathrm{m}^{3}\right)$ and higher unit price (350 euro $/ \mathrm{kg}$ ) is the most inadequate material for car frame construction and is classified in the lowest rank with respect to both mass saving and cost. 
Considering the above results, the five candidate materials have been classified concerning their mass saving and cost in table 2.

Table 2: Classification of the candidate materials with respect to normalized mass (N.M.) and normalized cost (N.C.).

\begin{tabular}{|l|l|l|}
\hline \multicolumn{2}{|c|}{ N.M. } & \multirow{2}{*}{ N.C. } \\
\hline Stiffness & Strength & \\
\hline CFRP & CFRP & CFRP \\
\hline Aluminum & Aluminum & Steel \\
\hline Steel & Steel & Nickel \\
\hline Nickel & Nickel & Aluminum \\
\hline Copper & Copper & Copper \\
\hline
\end{tabular}

\section{Conclusions}

In this work a quantitative analysis was performed to review five widely used materials with respect to their suitability for a cost effective light-weight car frame construction aiming at the minimization of fuel consumption. A simple techno-economical model was developed to obtain the interaction between material properties and material prices in order to make the optimum decision in the material selection. The analysis shows that the composite materials CFRPs result in lighter car frames with the same stiffness and strength (compared with the other candidate materials) with the minimum cost. More specifically, the classification of the five candidate materials with respect to their mass saving is CFRP, Aluminum, Steel, Nickel, Copper (for same stiffness and strength) while with respect to the cost saving the classification is CFRP, Steel, Nickel, Aluminum and Copper. However, due to CFRPs' inability to be recycled, a continuation of this study is required to answer if the total influence on the environment is beneficial or disadvantageous.

\section{References}

[1] Ashby, M.F. \& Jones, D.R.H., Engineering Materials I, Pergamon: Oxford, 1980.

[2] Koller, G., Risk Assessment and Decision Making in Business and Industry, Chapman \& Hall/CRC: London, 2005.

[3] Shapira, Z., Organizational Decision Making, Cambridge University Press: Cambridge, 2002.

[4] Drucker, P.F., Hammond, J., Keeney, R., Raiffa, H., Hayashi, A.M., Harvard Business Review on Decision Making, Harvard Business School: Harvard, 2001.

[5] Ingersoll, J.E., Theory of Financial Decision Making, Rowman \& Littlefield Publ., 1987.

[6] Mikhelson, I., Structural Engineering Formulas, Mc Graw Hill: New York, 2004. 